TRANSACTIONS OF THE

AMERICAN MATHEMATICAL SOCIETY

Volume 355, Number 2, Pages 609-618

S 0002-9947(02)03128-8

Article electronically published on September 6, 2002

\title{
HYPERPLANE ARRANGEMENTS AND LINEAR STRANDS IN RESOLUTIONS
}

\author{
IRENA PEEVA
}

\begin{abstract}
The cohomology ring of the complement of a central complex hyperplane arrangement is the well-studied Orlik-Solomon algebra. The homotopy group of the complement is interesting, complicated, and few results are known about it. We study the ranks for the lower central series of such a homotopy group via the linear strand of the minimal free resolution of the field C over the Orlik-Solomon algebra.
\end{abstract}

\section{INTRODUCTION}

In this paper, $\mathcal{A}$ stands for a central hyperplane arrangement of hyperplanes $H_{1}, \ldots, H_{n}$ in $\mathbf{C}^{l}$. The complement $X=\mathbf{C}^{l} \backslash \bigcup_{i=1}^{n} H_{i}$ has a complicated fundamental group $\pi_{1}(X)$. Let $Z=Z_{1}=\pi_{1}(X), Z_{2}, \ldots, Z_{i+1}=\left[Z_{i}, Z\right], \ldots$ be the lower central series and set $\varphi_{i}=\operatorname{rank}\left(Z_{i} / Z_{i+1}\right)$. Our goal is to obtain information (formulas, bounds, computational algorithms) about the ranks $\varphi_{i}$ in terms of some invariants of the algebra $\mathbf{H}^{*}(X ; \mathbf{C})$; this relates the homotopy and cohomology of $X$. The ranks $\varphi_{i}$ are studied through the generating function

$$
\prod_{j=1}^{\infty}\left(1-t^{j}\right)^{\varphi_{j}}
$$

Results of Kohno $[\mathrm{Ko}$ lead to Theorem 2.6. It translates the problem of computing (1.1) from Algebraic Topology into the entirely algebraic problem of describing the linear strand of the minimal free resolution of $\mathbf{C}$ over a certain algebra (which is a special type of a quotient of an exterior algebra and is related to the OrlikSolomon algebra $\left.\mathbf{H}^{*}(X ; \mathbf{C})\right)$. It also opens up the possibility to compute some numbers $\varphi_{j}$ by the computer algebra package MACAULAY 2 by D. Grayson and M. Stillman.

Theorem 2.7 provides a coefficientwise upper bound for (1.1).

For the arrangement $D_{3}^{1}$, the numbers $\varphi_{1}, \varphi_{2}, \varphi_{3}$ were computed in [FR]; furthermore, Björner and Ziegler [BZ, 4.1(1)] constructed a rooting for defining the ideal of the Orlik-Solomon algebra. In Theorem 3.10, we show for this arrangement that (1.1) is not equal to the Hilbert series of any standard graded algebra.

The main result obtained by Falk and Randell in [FR, which was reproved in [SY, Theorem 5.6], is that the Lower Central Series formula (3.1) holds for any supersolvable arrangement. In Section 4 we present a short proof, which makes

Received by the editors January 15, 1998 and, in revised form, December 21, 1998.

2000 Mathematics Subject Classification. Primary 13D02.

This work was partially supported by NSF. 
use of the Gröbner basis theory. The effect of having a quadratic Gröbner basis is explained in Lemma 3.6.

It was asked whether the Lower Central Series formula (3.1) can hold for a nonsupersolvable arrangement. Further, it was expected by Shelton and Yuzvinsky in SY, 6.4] that the formula holds for the nonsupersolvable arrangement of the affine plane of order 3. In Example 4.5 we disprove this. The referee noticed that Example 4.5 resolves the remark after Corollary 3.5 in $[\mathrm{Fa}$, since Example 4.5 provides a 2-determined arrangement that is not $K(\pi, 1)$.

It was first noted in $[\mathrm{SY}]$ that Koszul algebras are useful in the study of (1.1). We go further and introduce a new formula (3.4), which is a natural generalization of the Lower Central Series formula. In Section 5 we give examples when (3.4) holds. In this section we study subarrangements of $A_{l-1}$. By a result of Edelman and Reiner [ER, 3.3], a subarrangement of $A_{l-1}$ is free if and only if it is supersolvable. Using (3.4) we compute (1.1) for some nonfree subarrangements of $A_{l-1}$.

In view of (3.4), it is natural to ask whether (1.1) is always equal to the Hilbert function of some standard graded algebra. Theorem 3.10 shows that the answer is negative.

Examples 3.9 and 4.5 are computed using the computer algebra program LIEDIM by C. Löfwall.

\section{LINEAR STRANDS IN RESOLUTIONS}

We first recall three constructions, which will be related by Theorem 2.4, proved by Kohno. This theorem shows that the ranks in the lower central series of $\pi_{1}(X)$ can be expressed in terms of the generating function of the holonomy algebra; on the other hand, the holonomy algebra is nicely related to the Orlik-Solomon algebra:

Construction 2.1 (Holonomy algebra). The holonomy Lie algebra $G$ is the quotient of the universal Lie algebra $T^{\prime}$ on $H_{1}(X, \mathbf{C})$ over the ideal generated by the image of the comultiplication $H_{2}(X, \mathbf{C}) \rightarrow H_{1}(X, \mathbf{C}) \otimes H_{1}(X, \mathbf{C})$. The enveloping algebra $U$ of $G$ is called the holonomy algebra of $\mathcal{A}$.

Construction 2.2 (Orlik-Solomon algebra). A set $S \subset\{1, \ldots, n\}$ is called dependent if $\operatorname{rank}\left(\bigcap_{i \in S} H_{i}\right)<|S|$, where the rank is taken in the intersection lattice $L_{\mathcal{A}}$ of the arrangement. Furthermore, $S$ is a circuit if it is dependent and has minimal support among the dependent sets, and $S$ is a broken circuit if there is an $H_{i}$ such that $S \cup i$ is a circuit and $i>\max (S)$. Let $E$ be the exterior algebra over $\mathbf{C}$ on $n$ generators $x_{1}, \ldots, x_{n}$; this is a differential algebra with differential $d$ acting as $d\left(x_{i_{1}} \wedge \cdots \wedge x_{i_{s}}\right)=\sum_{j=1}^{s}(-1)^{j+1} x_{i_{1}} \wedge \cdots \wedge \hat{x}_{i_{j}} \wedge \cdots \wedge x_{i_{s}}$, (here $\hat{x}_{i_{j}}$ means that this variable is not present in the product). Let $I$ be the ideal in $E$ generated by $\{d$ (circuit) $\}$. Then $A=E / I$ is called the Orlik-Solomon algebra of $\mathcal{A}$. It is isomorphic to $H^{*}(X, \mathbf{C})$; cf. OT. The generating function $P(t)=$ $\sum \operatorname{dim}\left(H^{i}(X, \mathbf{C})\right) t^{i}$ is the Poincaré polynomial of $\mathcal{A}$.

In this paper, we will also consider the algebra $Q=E /\left\langle I_{2}\right\rangle$, where $I_{2}$ is the quadratic part of the ideal $I$. Thus, $\left\langle I_{2}\right\rangle$ is generated by $\{d(3$-circuit $)\}$ and we call $Q$ the 3 -circuit Orlik-Solomon algebra (here by a 3 -circuit we mean a circuit with three elements in its support). Then $A$ is a quotient of $Q$. Another algebra, which we will use, is $\bar{A}=A /\left(x_{1}, \ldots, x_{n}\right)^{3}=E /\left\langle I_{2}+\left(x_{1}, \ldots, x_{n}\right)^{3}\right\rangle=\mathbf{C}+A_{1}+A_{2}$; this is an Artinian algebra of height 2. 
Construction 2.3. [cf. [Ei, 17.22]] Let $T$ be the tensor algebra on the vector space $V=A_{1}=H_{1}(X, \mathbf{C})$ generated by the variables $x_{1}, \ldots, x_{n}$. Identify $(V \otimes V)^{*}$ with $V^{*} \otimes V^{*}$ by the rule $(a \otimes b)(v \otimes w)=a(v) b(w)$ for $a, b \in V^{*}, v, w \in V$. For a subspace $P \subset V \otimes V$, define the perpendicular space $P^{\perp}=\left\{a, \mid a \in V^{*} \otimes V^{*}, a(q)=\right.$ 0 for any $q \in P\}$. If $J$ is the ideal generated by $P$, then we denote by $J^{\perp}$ the ideal in $T\left(V^{*}\right)$ generated by $P^{\perp}$. If $R=T(V) / J$, then we set $R^{\perp}=T\left(V^{*}\right) / J^{\perp}$.

With the above notation, let $J$ be the ideal generated by quadrics, such that $Q=T(V) / J$; i.e.,

$$
J=\left\langle x_{i}^{2}, x_{i} x_{j}+x_{j} x_{i}, I_{2} \mid 1 \leq i, j \leq n\right\rangle .
$$

It was observed in [SY, Lemma 5.1] that $U=Q^{\perp}$, where $U$ is the holonomy algebra.

For a finitely generated graded $\mathbf{C}$-algebra $R$, we denote by $\operatorname{Hilb}_{R}(t)$ the generating Hilbert function $\sum_{i} \operatorname{dim}\left(R_{i}\right) t^{i}$.

Theorem 2.4 [Ko, 2.19, 2.21].

$$
\prod_{j=1}^{\infty}\left(1-t^{j}\right)^{-\varphi_{j}}=\sum_{s} \operatorname{dim}\left(U_{s}\right) t^{s}=\operatorname{Hilb}_{U}(t)
$$

where $U=Q^{\perp}$ is the holonomy algebra.

Construction 2.5. Let $R=E / M$ for some homogeneous ideal $M$ in $E$ and let

$$
\mathbf{F}: \ldots F_{i+1} \rightarrow F_{i} \rightarrow \ldots \rightarrow F_{1} \rightarrow F_{0} \rightarrow \mathbf{C}
$$

be the minimal free resolution of $\mathbf{C}$ over $R$. Furthermore, the resolution is graded by the total monomial degree; so $F_{i}=\bigoplus_{j \geq i} F_{i, j}$ (here the first grading is homological and the second one is monomial). The ranks $b_{i, j}=\operatorname{rank}\left(F_{i, j}\right)=\operatorname{dim}\left(\operatorname{Tor}_{i}^{R}(\mathbf{C}, \mathbf{C})_{j}\right)$ $=\operatorname{dim}\left(\operatorname{Ext}_{R}^{i}(\mathbf{C}, \mathbf{C})_{j}\right)$ are called the Betti numbers of $\mathbf{C}$ over $R$, and $b_{i, i}$ are called linear Betti numbers. The last name comes from the fact that the complex $\ldots \rightarrow$ $F_{i+1, i+1} \rightarrow F_{i, i} \rightarrow \ldots$ is indeed the linear part of the resolution $\mathbf{F}$, that is, the entries in the maps of the differential are linear forms. This linear part of $\mathbf{F}$ is called the linear strand.

The generating function $\mathrm{P}_{\mathbf{C}}^{R}(t, u)=\sum_{s} b_{s, p} t^{s} u^{p}$ is called the Poincaré series of $\mathbf{C}$ over $R$, and $\operatorname{linP}_{\mathbf{C}}^{R}(t)=\sum_{s} b_{s, s} t^{s}$ is called the linear Poincaré series of $\mathbf{C}$ over $R$.

Applying the above construction, we get that Theorem 2.4 leads to:

\section{Theorem 2.6.}

$$
\begin{aligned}
& \prod_{j=1}^{\infty}\left(1-t^{j}\right)^{-\varphi_{j}} \\
& \quad=\operatorname{linP}_{\mathbf{C}}^{Q}(t)=\operatorname{lin}_{\mathbf{C}}^{A}(t)=\operatorname{linP}_{\mathbf{C}}^{\bar{A}}(t) \\
& \quad=\frac{t \mathrm{P}_{\mathbf{C}}^{\bar{A}}(t)}{1+t}+\frac{1}{1+(1-n) t+\left(\operatorname{dim}\left(A_{2}\right)-n\right) t^{2}+\operatorname{dim}\left(A_{2}\right) t^{3}} .
\end{aligned}
$$

The theorem allows us to study the numbers $\varphi_{j}$ via either the linear Poincaré series over the Orlik-Solomon algebra $A$ (or over $Q$ ), or by the total Poincaré series over the short Artinian algebra $\bar{A}$. In the case $I_{2}=\emptyset$, the formula is simple:

$$
\prod_{j=1}^{\infty}\left(1-t^{j}\right)^{\varphi_{j}}=\frac{1}{1+n t} .
$$


Proof. By [Ei, 17.22], the algebra $Q^{\perp}$ is the subalgebra of $\operatorname{Ext}_{Q}^{*}(\mathbf{C}, \mathbf{C})$ generated by $\operatorname{Ext}_{Q}^{1}(\mathbf{C}, \mathbf{C})$, i.e., this is $\bigoplus \operatorname{Ext}_{Q}^{i}(\mathbf{C}, \mathbf{C})_{i}$. Thus, $\operatorname{dim}\left(Q_{i}^{\perp}\right)=b_{i, i}$. By Theorem 2.4, it follows that $\prod_{j=1}^{\infty}\left(1-t^{j}\right)^{-\varphi_{j}}=\sum_{s} b_{s, s} t^{s}$.

The linear strand is not affected by adding or removing generators of degree $\geq 3$ from the ideal that we mod out. Thus, the linear strand is the same when we resolve over $A$, or over $Q$, or over $\bar{A}$.

For the algebra $\bar{A}$, we have $\operatorname{dim}\left(\bar{A}_{0}\right)=1, \operatorname{dim}\left(\bar{A}_{1}\right)=n, \operatorname{dim}\left(\bar{A}_{2}\right)=\operatorname{dim}\left(A_{2}\right)$, and $\operatorname{dim}\left(\bar{A}_{i}\right)=0$ for $i \geq 3$. The fact that the Euler characteristic of $\mathbf{F}$ vanishes is expressed in the formula

$$
\left(1-n t+\operatorname{dim}\left(\bar{A}_{2}\right) t^{2}\right)\left(\operatorname{lin}_{\mathbf{C}}^{\bar{A}}(t)-t\left(\mathrm{P}_{\mathbf{C}}^{\bar{A}}(t)-\operatorname{lin}_{\mathbf{C}}^{\bar{A}}(t)\right)\right)=1 .
$$

This leads to

$$
\operatorname{lin}_{\mathbf{C}}^{\bar{A}}(t)=\frac{t \mathrm{P}_{\mathbf{C}}^{\bar{A}}(t)}{1+t}+\frac{1}{1+(1-n) t+\left(\operatorname{dim}\left(A_{2}\right)-n\right) t^{2}+\operatorname{dim}\left(A_{2}\right) t^{3}} .
$$

An important application of Theorem 2.6 is that it opens up the possibility to compute $\varphi_{j}$ 's in concrete examples by computer. The Betti numbers $b_{s, s}$ could be computed by the computer algebra package MACAULAY 2 by D. Grayson and M. Stillman.

Theorem 2.7. Let $B$ be the ideal in $E$ generated by the quadratic broken circuits. Then

$$
\prod_{j=1}^{\infty}\left(1-t^{j}\right)^{-\varphi_{j}} \ll \frac{1}{\operatorname{Hilb}_{E / B}(-t)},
$$

where $\ll$ means coefficientwise inequality of power series.

We briefly explain the relation between $B$ and $\left\langle I_{2}\right\rangle$. Let $G$ be the initial ideal of $\left\langle I_{2}\right\rangle$ with respect to the lexicographic order. Then there exists a flat family connecting $E / G$ and $Q=E /\left\langle I_{2}\right\rangle$; in particular, the two algebras have the same Hilbert function. On the other hand, the ideal $B$ generated by the quadratic broken circuits equals $\left\langle G_{2}\right\rangle$; so we have a surjection $E / B \rightarrow E / G$. Thus, the Hilbert function of $E / B$ majorates the one of $Q$.

Proof. Let $G$ be the initial ideal of $\left\langle I_{2}\right\rangle$ with respect to the lexicographic order. We will show that the following relations hold:

$$
\begin{aligned}
& \prod_{j=1}^{\infty}\left(1-t^{j}\right)^{-\varphi_{j}}=\operatorname{lin}_{k}^{Q}(t) \\
& \ll \operatorname{linP}_{\mathbf{C}}^{E / G}(t)=\operatorname{lin}_{\mathbf{C}}^{E /\left\langle G_{2}\right\rangle}(t)=\operatorname{lin}_{\mathbf{C}}^{E / B}(t)=\frac{1}{\operatorname{Hilb}_{E / B}(-t)} .
\end{aligned}
$$

The first equality is from Theorem 2.6. The second inequality follows from the Gröbner basis theory (by a standard deformation argument; cf. Ei, Theorem 15.17]). The third equality holds because the linear strand in the minimal free resolution of $\mathbf{C}$ does not change if we remove the nonquadratic generators of $G$. The fourth equality comes from the fact that the ideal $B$ generated by the quadratic broken circuits is $\left\langle G_{2}\right\rangle$. The last equality holds because $B$ is a monomial quadratic ideal and $E / B$ is Koszul by $[\mathrm{Fr}]$. 


\section{Lower Central Series formulas}

The Lower Central Series formula (or LCS formula for short) is a special formula, which has received a lot of attention in $[\mathrm{FR},[\mathrm{Ko},[\mathrm{SY}]$. It states that

$$
\prod_{j=1}^{\infty}\left(1-t^{j}\right)^{\varphi_{j}}=\operatorname{Hilb}_{A}(-t) .
$$

It was first noted by Shelton and Yuzvinsky in [SY] (see also Theorem 2.6) that the formula holds if and only if the algebra $A$ is Koszul. Koszulness means that there are only linear entries in the matrices of the maps in the minimal free resolution of $\mathbf{C}$ over $A$, i.e., $b_{i j}=0$ for $i \neq j$. The fact that the Euler characteristic of this resolution vanishes shows that Koszulness is equivalent to $\operatorname{linP}_{\mathbf{C}}^{A}(t)=\mathrm{P}_{\mathbf{C}}^{A}(t, 1)=\frac{1}{\operatorname{Hilb}_{A}(-t)}$. This raises the question:

\section{Question 3.2. When is A Koszul?}

Note that a necessary condition for $A$ being Koszul is that $I$ is generated by quadrics. One simple sufficient condition for this to hold is the following:

Lemma 3.3. The ideal $I$ is generated by quadrics if every circuit $S$ with $|S| \geq 4$ satisfies the following condition: there exists a number $j_{S}$ and $S=S_{1} \cup S_{2}$, so that $S_{1} \cup\left\{j_{S}\right\}$ and $S_{2} \cup\left\{j_{S}\right\}$ are circuits and $S_{1} \cap S_{2}=\emptyset$.

Proof. Set $f_{1}=\bigwedge_{i \in S_{1}} x_{i}$ and $f_{2}=\bigwedge_{i \in S_{2}} x_{i}$. Then $d\left(f_{1} \wedge x_{j_{s}}\right)=d\left(f_{1}\right) \wedge x_{j_{s}}-$ $(-1)^{\left|S_{1}\right|} f_{1}$ and $d\left(x_{j_{s}} \wedge f_{2}\right)=x_{j_{s}} \wedge d\left(f_{2}\right)-f_{2}$. Multiplying the first equality by $d\left(f_{2}\right)$ and the second by $-d\left(f_{1}\right)$ we get

$$
\begin{aligned}
d\left(f_{1} \wedge x_{j_{s}}\right) \wedge d\left(f_{2}\right) & =d\left(f_{1}\right) \wedge x_{j_{s}} \wedge d\left(f_{2}\right)-(-1)^{\left|S_{1}\right|} f_{1} \wedge d\left(f_{2}\right), \\
-d\left(f_{1}\right) \wedge d\left(x_{j_{s}} \wedge f_{2}\right) & =-d\left(f_{1}\right) \wedge x_{j_{s}} \wedge d\left(f_{2}\right)+d\left(f_{1}\right) \wedge f_{2} .
\end{aligned}
$$

Adding the above equalities, we obtain

$$
d\left(f_{1}\right) \wedge f_{2}-(-1)^{\left|S_{1}\right|} f_{1} \wedge d\left(f_{2}\right)=d\left(f_{1} \wedge x_{j_{s}}\right) \wedge d\left(f_{2}\right)-d\left(f_{1}\right) \wedge d\left(x_{j_{s}} \wedge f_{2}\right),
$$

where the left-hand side equals $d\left(f_{1} \wedge f_{2}\right)$. Since $S_{1} \cup\left\{j_{S}\right\}$ and $S_{2} \cup\left\{j_{S}\right\}$ are circuits, we conclude that the relation $d\left(f_{1} \wedge f_{2}\right)$ is generated by relations of lower degrees.

If $I \neq\left\langle I_{2}\right\rangle$, then it is natural to study $Q$. If $Q$ is Koszul, then a formula similar to the LCS holds:

$$
\prod_{j=1}^{\infty}\left(1-t^{j}\right)^{\varphi_{j}}=\operatorname{Hilb}_{Q}(-t)
$$

We call this a Generalized Lower Central Series formula (or GLCS formula for short). This formula holds if and only if the algebra $Q$ is Koszul (see Theorem 2.6). Thus, a natural generalization of the above question is:

Question 3.5. When is Q Koszul?

The most efficient technique in commutative algebra for answering questions such as 3.2 and 3.5 is to show that the algebra possesses a quadratic Gröbner basis. Gröbner basis theory works over exterior algebras $\underline{\mathrm{AHH}}$. We exploit the wellknown property that a quadratic Gröbner basis implies Koszulness. This property can be proved by a standard deformation argument; cf. [Ei, Ch.15]. In our case, the property implies that: 
Lemma 3.6. If I has a quadratic Gröbner basis, then the Lower Central Series formula (3.1) holds. If $\left\langle I_{2}\right\rangle$ has a quadratic Gröbner basis, then the formula (3.4) holds.

Next we give a simple example, which clearly demonstrates that $s$-circuits for $s \geq 4$ are irrelevant to our goals and that formula (3.4) and Lemma 3.6 could be very useful:

Example 3.7. Consider the graphic arrangement $x=y, y=z, z=u, u=v, z=$ $v, u=x$. The only 3 -circuit is $\{3,4,5\}$. Thus, in this case, $\left\langle I_{2}\right\rangle$ is generated by the quadric $x_{3} x_{4}-x_{3} x_{5}+x_{4} x_{5}$. Surely, this quadric is a Gröbner basis. Therefore, by Lemma 3.6, we have that the formula (3.4) holds:

$$
\prod_{j=1}^{\infty}\left(1-t^{j}\right)^{\varphi_{j}}=\operatorname{Hilb}_{Q}(-t)=1-6 t+14 t^{2}-16 t^{3}+9 t^{4}-2 t^{5} .
$$

Theorem 4.3 shows when $I$ has a quadratic Gröbner basis. The next question is open:

Question 3.8. When does $Q$ have a quadratic Gröbner basis?

We remark that the behavior of the linear strand could be very complex and formulas of the type (3.1) and (3.4) (even if we change the algebra appearing in the formula) are not sufficient to express the possible linear Poincare series. This is illustrated next.

Example 3.9. Consider the arrangement $z=0, z=x, z=-x, y=z, y=-z, x=$ $y, x=-y$ in $\mathbf{C}^{3}$ which is free. This is $D_{3}^{1}$ (non-Fano matroid). The Poincaré polynomial of $A$ is $(1+t)(1+3 t)^{2}$. In this case also [BZ, 4.1(1)] provides a quadratic rooting for $I$. However, the ideal $I$ is not generated by quadratic forms. In this case, the behavior of the linear strand is quite complex. The 3-circuits are

$$
\{1,2,3\},\{1,4,5\},\{2,4,6\},\{2,5,7\},\{3,5,6\},\{3,4,7\} .
$$

Computing by the computer algebra program LIEDIM, we find the first Betti numbers $b_{s, s}$ for $0 \leq s \leq 5$ : they are 1,7,34,143,560,2108,7753. By Theorem 2.6,

$$
\prod_{j=1}^{\infty}\left(1-t^{j}\right)^{-\varphi_{j}}=1+7 t+34 t^{2}+143 t^{3}+560 t^{4}+2108 t^{5}+7753 t^{6}+O\left(t^{7}\right)
$$

so we can compute the first numbers $\varphi_{j}$. We remark that $\varphi_{1}, \varphi_{2}, \varphi_{3}$ are computed in FR.

Theorem 3.10. For $D_{3}^{1}$ there does not exist a standard graded algebra $N$ such that

$$
\prod_{j=1}^{\infty}\left(1-t^{j}\right)^{\varphi_{j}}=\operatorname{Hilb}_{N}(-t) .
$$

Proof. Assume the opposite. Write

$$
\operatorname{Hilb}_{N}(-t)=1-7 t+\alpha t^{2}-\beta t^{3}+\gamma t^{4}-\mu t^{5}+\eta t^{6}+f,
$$

where $\alpha, \beta, \gamma, \mu, \eta$ are nonnegative integers and $f \in t^{7}(\mathbf{N}[[t]])$. Using the first six Betti numbers from Example 3.9, we obtain the relation

$$
\begin{aligned}
1+7 t & +34 t^{2}+143 t^{3}+560 t^{4}+2108 t^{5}+7753 t^{6}+O\left(t^{7}\right) \\
& =\frac{1}{1-7 t+\alpha t^{2}-\beta t^{3}+\gamma t^{4}-\mu t^{5}+\eta t^{6}-f(-t)},
\end{aligned}
$$

which implies $\alpha=15, \beta=10, \gamma=1, \mu=0$ and $\eta=-1$. This is a contradiction. 


\section{Supersolvable HyPERPLANE ARRANGEMENTS}

Theorem 4.1 (cf. Bj]). The broken circuits generate the initial ideal of I with respect to the lexicographic order $x_{1} \succ x_{2} \succ \cdots \succ x_{n}$.

Proposition 4.2. Let $\prec$ be any monomial order with $x_{1} \succ x_{2} \succ \cdots \succ x_{n}$ and let $\prec_{\text {lex }}$ be the lexicographic order, which orders the variables in the same way. Then $i n_{\prec}(I)=i n_{\prec_{\text {lex }}}(I)$.

Proof. Let $p$ be a circuit. Then any two terms in $d(p)$ have the forms $q \wedge x_{i}$ and $q \wedge x_{j}$. Therefore, $q \wedge x_{i} \prec q \wedge x_{j}$ exactly when $q \wedge x_{i} \prec_{\text {lex }} q \wedge x_{j}$. Hence $i n_{\prec}(p)=i n_{\prec_{\text {lex }}}(p)$. But by Theorem 4.1, the set $\{d$ (circuit) $\}$ is a Gröbner basis with respect to the lexicographic order; so we can apply [Ei, 15.16].

Theorem 4.3. There exists a quadratic Gröbner basis for $I$ if and only if $\mathcal{A}$ is supersolvable.

Proof. By Proposition 4.2, the ideal $I$ possesses a quadratic Gröbner basis exactly when there exists a quadratic set of minimal broken circuits. By [BZ] this is equivalent to $\mathcal{A}$ being supersolvable.

Corollary 4.4. If $\mathcal{A}$ is supersolvable, then the LCS formula (3.1) holds.

Proof. Apply Lemma 3.6 and Theorem 4.3.

It is an open question whether the LCS formula (3.1) can hold for a nonsupersolvable arrangement. The following example is from [Zi]; it was expected in [SY] Example 6.4] that (3.1) holds in this case.

Example 4.5. The Lower Central Series formula (3.1) does not hold for the nonsupersolvable arrangement corresponding to the affine plane of order 3.

Proof. The 3-circuits are

$$
\begin{aligned}
& \{1,2,3\},\{4,5,6\},\{7,8,9\},\{1,4,7\},\{2,5,8\},\{3,6,9\}, \\
& \{1,5,9\},\{2,6,7\},\{3,4,8\},\{1,6,8\},\{2,4,9\},\{3,5,7\} .
\end{aligned}
$$

The ideal $I$ is generated by quadratic forms (see [SY] Example 6.4]). The matroid is very symmetric, but not supersolvable. Therefore, by Theorem 4.3, there exists no quadratic Gröbner basis of $I$. Björner and Ziegler constructed in [BZ, 4.1.(4)] a quadratic rooting for $I$. Equivalently, they constructed the monomial ideal

$$
M=\left\langle x_{1} x_{3}, x_{1} x_{7}, x_{1} x_{9}, x_{2} x_{4}, x_{2} x_{6}, x_{2} x_{8}, x_{3} x_{7}, x_{3} x_{9}, x_{4} x_{6}, x_{4} x_{8}, x_{7} x_{9}, x_{6} x_{8}\right\rangle,
$$

for which the Hilbert function of $E / M$ is the same as for $A$. However, the ideal $M$ does not come as an initial ideal and no deformation can be applied using it. Computing by LIEDIM we obtain the Betti numbers $b_{1,1}=9, b_{2,2}=57, b_{3,3}=$ $313, b_{4,4}=1593$, which is as if it were Koszul; but then $b_{4,5}=3$. Thus, $A$ is not Koszul, and equivalently, the LCS formula (3.1) does not hold.

\section{Quadratic Gröbner basis And Graphic ARrangements}

Next we present a technical local condition, which guarantees that there exists a quadratic Gröbner basis. After that we will apply this condition to graphic arrangements. 
Construction 5.1. Let $x_{1}, \ldots, x_{6}$ be variables in $E$ corresponding to hyperplanes in $\mathcal{A}$. Let $(i, j, p, q, r, s)$ be a permutation of $(1,2,3,4,5,6)$; we denote by $\prec_{i j p q r s}$ a lexicographic order in $E$ for which the variables $x_{1}, \ldots, x_{6}$ are ordered so that $x_{1}$ is $i$ th, $x_{2}$ is $j$ th, etc.; for example, $\prec_{315426}$ means that $x_{2} \succ x_{5} \succ x_{1} \succ x_{4} \succ x_{3} \succ x_{6}$. We say that a lexicographic order is 135,234-quadratic if either

(1) $\{1,3,5\}$ or $\{2,3,4\}$ is not a circuit; or

(2) $x_{3}$ is smaller than $x_{1}$ and $x_{5}$; or

(3) $x_{3}$ is smaller than $x_{2}$ and $x_{4}$, or else there exists a hyperplane $H_{6}$, such that $\{1,2,6\}$ and $\{4,5,6\}$ are circuits and one of the following conditions is satisfied:

(4) the variables $x_{1}, x_{2}, x_{3}$ are bigger than the variables $x_{4}, x_{5}, x_{6}$;

(5) the order is $\prec_{412356}$;

(6) the order is $\prec_{412365}$;

(7) the order is $\prec_{124563}$;

(8) the order is $\prec 124653$;

(9) the order is $\prec_{241536}$;

(10) the order is $\prec_{241635}$;

(11) the order is $\prec_{315462}$.

Lemma 5.2. Let $\prec$ be a lexicographic order on $E$ such that it is ipr, jpq-quadratic for any choice of different indices $i, j, p, q, r$. Then $Q$ has a quadratic Gröbner basis.

Proof. We want to show that the elements $\{d(3$-circuit $)\}$ form a Gröbner basis. Consider two elements of this form which form a Gröbner pair, i.e., whose initial terms have a common variable. After renumbering, we may assume that these elements are $d\left(x_{1} x_{3} x_{5}\right)$ and $d\left(x_{2} x_{3} x_{4}\right)$. In cases (1), (2), and (3) in Construction 5.1, we do not get a Gröbner pair. In the rest of the cases, direct computation using Buchberger's criterion (or computing by MACAULAY 2) shows that $d\left(x_{1} x_{3} x_{5}\right), d\left(x_{2} x_{3} x_{4}\right), d\left(x_{1} x_{2} x_{6}\right)$, and $d\left(x_{4} x_{5} x_{6}\right)$ form a quadratic Gröbner basis for the ideal that they generate.

Next, we will apply Lemma 5.2 to graphic arrangements. We assume that all graphs are simple, that is, there could be only a single edge between any two vertices and there are no loops. Recall that a graph $G$ defines a graphic arrangement $\mathcal{A}_{G}$ in the following way: Let the vertices of the graph be labeled by $1, \ldots, l$. Denote by $u_{1}, \ldots, u_{l}$ the coordinates in $\mathbf{C}^{l}$. Then the hyperplane $u_{i}=u_{j}$ is in $\mathcal{A}_{G}$ exactly when there is an edge between $i$ and $j$ in $G$. Graphic arrangements are exactly the subarrangements of $A_{l-1}$.

Theorem 5.3. Let $\mathcal{A}_{G}$ be the graphic arrangement corresponding to a graph $G$. Let the vertices of $G$ admit a labeling with $\{1, \ldots, l\}$ for which the following condition is satisfied: If $\{i, j\}$ is a common edge of two triangles $\{i, j, p\}$ and $\{i, j, q\}$, and $\{i, j\}$ contains the biggest vertex for each of the triangles, then $G$ also contains the edge $\{p, q\}$. Then formula (3.4) holds.

Proof. If $\{i, j\}$ is an edge in $G$, then denote by $x_{i j}$ the variable in $E$ corresponding to the hyperplane $u_{i}=u_{j}$. Then we define a lexicographic order on $E$ by

$$
x_{i j} \prec x_{p q} \text { if }\{p, q\} \prec_{\text {lex }}\{i, j\},
$$

where we compare the two sets $\{i, j\},\{p, q\}$ lexicographically. For example, $x_{24} \succ$ $x_{15}$. This order is well known and exploited. We will apply Lemma 5.2. Consider 
an edge $\{i, j\}$. Three cases are possible: If $\{i, j\}$ is not shared by two triangles, then case (1) from Construction 5.1 holds. Let $\{i, j\}$ be the common edge of two triangles. If $\{i, j\}$ does not contain the biggest vertex from each triangle, then case (2) or (3) from Construction 5.1 holds. Let $i$ be the biggest vertex in each of the triangles. Then $\{p, q\}$ is an edge by assumption. Also, the variables $x_{i j}, x_{i p}, x_{i q}$ are bigger than the variables $x_{p q}, x_{p j}, x_{q j}$. Thus, case (4) from Construction 5.1 holds. By Lemma 5.2, we have that $Q$ has a quadratic Gröbner basis. Now apply Lemma 3.6.

Note that the above criterion involves only the triangles in the graph, while the other circuits are irrelevant. Also, if $I$ is generated by quadratic elements and the condition in Theorem 5.3 is satisfied, then $I$ has a quadratic Gröbner basis; so the arrangement is supersolvable.

Example 5.4. By Theorem 5.3, it follows that formula (3.4) holds for the graphic arrangement of the graph with seven vertices and edges

$$
\begin{aligned}
& \{1,2\},\{1,3\},\{2,3\},\{2,4\},\{3,4\},\{3,5\}, \\
& \{3,6\},\{3,7\},\{4,5\},\{5,6\},\{5,7\},\{6,7\} .
\end{aligned}
$$

Thus, we get

$$
\prod_{j=1}^{\infty}\left(1-t^{j}\right)^{\varphi_{j}}=\operatorname{Hilb}_{Q}(-t)=1-12 t+59 t^{2}-152 t^{3}+216 t^{4}-160 t^{5}+48 t^{6} .
$$

Note that by [ER, Theorem 3.3], this arrangement is not free since it is not supersolvable.

\section{ACKNOWLEDGMENTS}

Special thanks go to Günter Ziegler for inspiring and very helpful conversations. I am very grateful to Clas Löfwall, who made computations for Examples 3.9 and 4.5 using his computer algebra program LIEDIM. Also, I thank Vic Reiner for helpful discussions on Section 5.

\section{REFERENCES}

[AHH] A. Aramova, J. Herzog, and T. Hibi, Gotzmann theorems for exterior algebras and combinatorics, J. Algebra 191 (1997), 174-211. MR 98c:13025

[Bj] A. Björner, The homology and shellability of matroids and geometric lattices, in Matroid Applications (ed.: N. White) Cambridge University Press, 1992. MR 94a:52030

[BZ] A. Björner and G. Ziegler, Broken circuit complexes: Factorizations and generalizations, J. Combin. Theory Series B 51 (1991), 96-126. MR 92b:52027

[ER] P. Edelman and V. Reiner, Free hyperplane arrangements between $A_{n-1}$ and $B_{n}$, Math. Z. 215 (1994), 347-365. MR 95b:52021

[Ei] D. Eisenbud, Commutative Algebra with a View Towards Algebraic Geometry, SpringerVerlag, New York, 1995. MR 97a:13001

[Fa] M. Falk, The minimal model of an arrangement of hyperplanes, Trans. Amer. Math. Soc. 309 (1988), 543-556. MR 89d:32024

[Fr] R. Fröberg, Determination of a class of Poincaré series, Math. Scand. 37 (1975), 29-39. MR 53:8057

[FR] M. Falk and R. Randell, The lower central series of a fiber-type arrangement, Invent. Math. 82 (1985), 77-88. MR 87c:32015b

[Ko] T. Kohno, Série de Poincaré-Koszul associée aux groupes de tresses pures, Invent. Math. 82 (1985), 57-75. MR 87c:32015a

[OT] P. Orlik and H. Terao, Arrangements of hyperplanes, Springer-Verlag, New York, 1992. MR 94e:52014 
[SY] B. Shelton and S. Yuzvinsky, Koszul algebras from graphs and hyperplane arrangements, J. London Math. Soc. 56 (1997), 477-490. MR 99c:16044

[Zi] G. Ziegler, Matroid representations and free arrangements, Trans. Amer. Math. Soc. 320 (1990), 525-541. MR 90k:32045

Department of Mathematics, Massachusetts Institute of Technology, Cambridge, MASSACHUSETTS 02139

Current address: Department of Mathematics, Cornell University, Malott Hall, Ithaca, New York 14853-4201 